\title{
L'évolution de la contamination du lait par les insecticides organochlorés entre 1970 et $19722^{*}$
}

\author{
par \\ H. MAHIEU \\ Ingénieur agronome E.N.S.A. Rennes à la Section Lait de l'Institut \\ Technique de l'Elevage Bovin
}

\section{Rés u mé}

L'enquête entreprise par la Section Lait de l'Institut Technique de l'Elevage Bovin (I.T.E.B.) avait pour but de définir les processus les plus fréquents par lesquels les pesticides organochlorés peuvent contaminer le lait à des taux excédant les limites généralement admises.

Cette enquête a eu lieu au niveau de l'exploitation agricole. Elle n'a pas de prétention statistique, mais se contente de déterminer par l'étude approfondie d'un nombre de cas limités, mais bien répartis sur l'ensemble du territoire français, comment et pourquoi un lait peut se trouver contaminé.

A la suite d'interdictions successives auxquelles l'enquête n'est d'ailleurs pas étrangère, plusieurs causes de contamination sont passées au second plan (blanchiment des étables...) laissant en 1972 comme cause de contamination principale : l'alimentation.

L'étude de la contamination aliment par aliment permet d'énoncer quelques remarques quant au bien-fondé de l'interdiction de l'utilisation des cyclodiènes en agriculture.

Quant à l'évolution de la contamination du lait... Si on constate une diminution importante des résidus de $\mathrm{HCH}$ d'un hiver sur l'autre, certaines régions sont loin de répondre aux tolérances de nos partenaires commerciaux et cela, du fait de la rémanence de ces produits. En ce qui concerne les résidus d'heptachlore-époxyde, l'escalade de la contamination fut catastrophique surtout dans les

(*) Communication faite devant la Société Française de Phytiatrie et Phytopharmacie, le 16 janvier 1974, à l'Institut National Agronomique, 16, rue ClaudeBernard - 75005 Paris. 
régions de cultures industrielles ; ceci correspondrait à la rétrocession de pulpes de betteraves contaminées pour l'alimentation des animaux.

\section{I. - INTRODUCTION}

L'enquête entreprise par l'I.T.E.B. avait pour but de définir les processus les plus fréquents par lesquels les pesticides organochlorés peuvent contaminer le lait à des taux excédant les limites généralement admises.

Cette enquête a eu lieu au niveau de l'exploitation agricole. Elle n'a pas de prétention statistique mais se contente de déterminer par l'étude approfondie d'un nombre de cas limités mais bien répartis sur l'ensemble du territoire français, comment et pourquoi un lait peut se trouver contaminé.

Les résultats de ce travail démontrent cependant qu'une large part des causes de contamination que l'on rencontre, s'étend forcément à l'ensemble des exploitations d'une même zone : ceci est le cas des produits de blanchiment des étables, des aliments du commerce qui ont pu être incriminés, et dont l'usage n'a pas été la seule exclusivité du producteur.

De ce fait, la plupart des conclusions de cette enquête sont généralisables à l'ensemble de la France. Elles peuvent donc contribuer à définir une politique.

\section{II. - MODALITES DE L'ENQUETE}

\section{a) Choix des exploitations}

Les exploitations choisies répondent à deux critères :

- le lait qu'elles livrent à la laiterie est contaminé par des résidus de pesticides organochlorés,

- l'exploitation possède suffisamment d'enregistrements sur son fonctionnement pour qu'il soit possible de pousser toutes les investigations nécessaires à la recherche des causes de contamination.

\section{b) Echantillonnage}

L'échantillon comprend 89 exploitations laitières réparties sur 30 départements et choisies dans les tournées de 15 laiteries ou groupes de laiteries:

- 82 producteurs de lait de vache répartis selon les régions laitières,

-7 producteurs de lait de brebis. 


\section{c) Substances recherchées}

Les substances organochlorées recherchées sont :

- $\mathrm{HCH}$ isomères $\alpha, \beta$ et $\gamma$.

- HCB et les différents corps sortant à ce niveau.

- Aldrine et dieldrine.

- Heptachlore et heptachlore-époxyde.

- DDT, DDD et DDE.

Nous citons le HCB et le DDT seulement pour mémoire car nous n'avons décelé la présence de leurs résidus que très peu souvent.

\section{d) Déroulement de l'enquête}

Lors de la pré-enquête, un questionnaire a été rempli chez chaque producteur. Puis, successivement, dans chacune des exploitations, quatre passages ont été effectués : trois en 1971 (hiver, juin et septembre) et un dernier en mars 1972. A chaque passage, étaient prélevés simultanément : un échantillon de lait et un échantillon de chaque aliment distribué aux animaux. L'analyse de tous ces échantillons a été faite systématiquement*.

En général, aucune vulgarisation n'a eu lieu qui aurait eu pour effet de réduire la contamination d'un passage à l'autre. Sauf dans quelques cas isolés, les variations saisonnières de la teneur en résidus de pesticides organochlorés ne sont ainsi pas modifiées par un changement d'attitude du producteur à l'égard de ce problème.

Ainsi, cette étude permet non seulement de mieux définir les causes de contamination du lait par des résidus de pesticides organochlorés mais encore, elle permet, à partir de renseignements accumulés, de constater l'évolution de la contamination des laits durant la période 1970-1972 et de se faire une idée de son évolution dans le futur.

\section{III. - LES SOURCES DE CONTAMINATION}

Sans développer les conclusions du rapport sur les causes de contamination (cf. liste des publications en fin d'exposé), nous pouvons dire que la contamination des laits par des résidus de pesticides organochlorés au niveau de l'exploitation, s'effectue par deux voies différentes (fig. 1) :

\footnotetext{
* Laboratoire de Phytopharmacie du C.N.R.A. à Versailles et Laboratoire d'Indus-
} trie laitière de l'E.N.S.I.A.A. à Douai. 


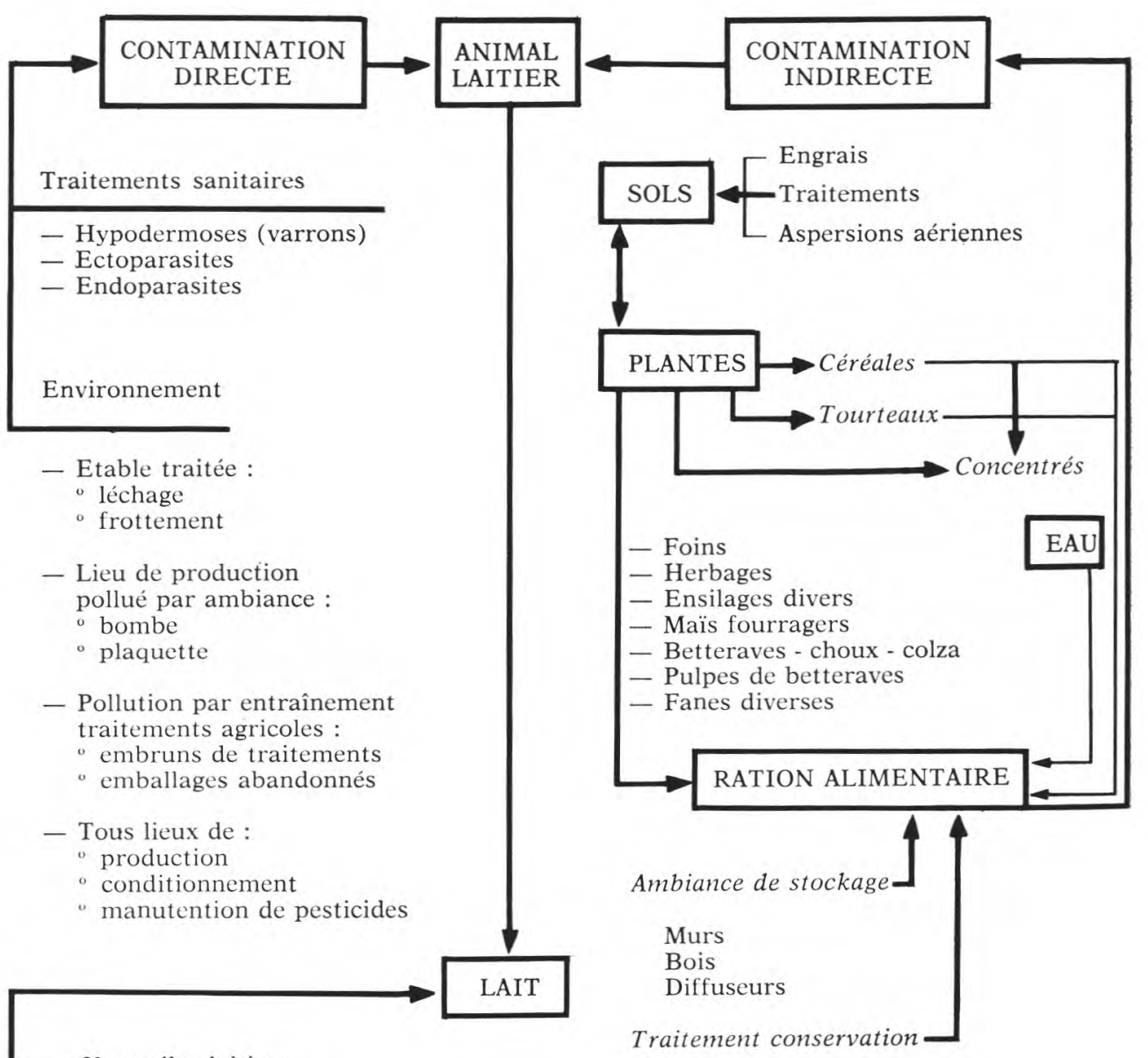

Ustensiles laitiers

Salle de collecte

Salle de transformation

ou de conditionnement en usine

fig. 1

Voies et origines de la contamination du lait

- par des produits ou des pratiques venus de l'extérieur dont le producteur ne peut absolument pas être tenu pour responsable, n'ayant aucun moyen de contrôle :

o traitements vétérinaires,

- traitements des locaux par des organismes spécialisés,

- achats de céréales, de tourteaux, d'aliments concentrés,

- rétrocession de pulpes de betteraves sucrières, 
TABLEAU 1. - Données sur la correspondance entre la contamination des aliments et celle du lait

\begin{tabular}{|c|c|c|c|c|c|c|}
\hline \multirow{4}{*}{$\begin{array}{c}\text { Substances } \\
\text { organochlorées } \\
\text { étudiées }\end{array}$} & \multirow{4}{*}{$\begin{array}{l}\text { Contamination } \\
\text { de } \\
\text { l'alimentation }\end{array}$} & \multirow{4}{*}{$\begin{array}{l}\text { Contamination } \\
\text { correspondante } \\
\text { du lait }\end{array}$} & \multicolumn{4}{|c|}{$\begin{array}{l}\text { Niveau maximum de contamination pour les catégories } \\
\text { d'aliments suivants }\end{array}$} \\
\hline & & & $1^{\mathrm{e}}$ catégorie & $2^{e}$ catégorie & \multicolumn{2}{|c|}{$3^{\text {e }}$ catégorie } \\
\hline & & & $\begin{array}{c}\text { Concentrés } \\
\text { Céréales } \\
\text { Tourteau } \\
\text { Pulpe (sèche) }\end{array}$ & $\begin{array}{c}\text { Foins } \\
\text { et } \\
\text { pailles } \\
\text { diverses }\end{array}$ & \multirow{2}{*}{\multicolumn{2}{|c|}{$\begin{array}{c}\text { Racines de betteraves } \\
\text { Choux - Colza - Collets } \\
\text { Prairies diverses } \\
\text { Ensilages divers } \\
\text { Ensilages de maïs } \\
\text { Autres fourrages grossiers } \\
\text { Cons. journ. } 10 \mathrm{~kg}\end{array}$}} \\
\hline & & & Cons. journ. $3 \mathrm{~kg}$ & Cons. journ. $7 \mathrm{~kg}$ & & \\
\hline $\mathrm{ppm}=$ & $\begin{array}{c}\text { mg dans la ration } \\
\text { totale } \\
\text { (MS journalière*) }^{*}\end{array}$ & $\underset{\text { grasse }}{\mathrm{mg} / \mathrm{kg} \text { de matière }}$ & $\mathrm{mg} / \mathrm{kg}$ d'aliment & $\mathrm{mg} / \mathrm{kg}$ d'aliment & $\begin{array}{l}\mathrm{mg} / \mathrm{kg} \\
\text { d'aliment }\end{array}$ & $\begin{array}{l}\mathrm{mg} / \mathrm{kg} \text { de } \\
\text { matière sèche }\end{array}$ \\
\hline$\alpha \mathrm{HCH}$ & 0,080 & 0,10 & 0,030 & 0,015 & 0,010 & 0,030 à 0,100 \\
\hline$\beta \mathrm{HCH}$ & 0,080 & 0,10 & 0,030 & 0,015 & 0,010 & 0,030 à 0,100 \\
\hline Lindane & 0,800 & 0,10 & 0,300 & 0,150 & 0,100 & 0,300 à 1,000 \\
\hline $\begin{array}{l}\text { Heptachlore- } \\
\text { époxyde }\end{array}$ & 0,040 & 0,10 & 0,010 & 0,005 & 0,005 & 0,015 à 0,050 \\
\hline Dieldrine & 0,050 & 0,10 & 0,010 & 0,005 & 0,005 & 0,015 à 0,050 \\
\hline DDT & 0,800 & 0,10 & 0,300 & 0,150 & 0,100 & 0,300 à 1,000 \\
\hline
\end{tabular}

* Sur la base de $60 \mathrm{~kg}$ de poids frais par jour.

** Production moyenne journalière de matière grasse : $484 \mathrm{~g}$. 
TABLEAU 2. - Contamination des diverses catégories d'aliments

(importance de la contamination globale et participation de chacun des insecticides organochlorés à cette contamination)

\begin{tabular}{|c|c|c|c|c|c|c|c|c|c|c|}
\hline & \multirow{2}{*}{ Aliments } & & \multirow{2}{*}{$\begin{array}{c}\text { Nombre } \\
\text { total } \\
\text { d'échantillons }\end{array}$} & \multirow{2}{*}{$\begin{array}{l}\text { Nombre* } \\
\text { d'échantillons } \\
\text { contaminés }\end{array}$} & \multicolumn{6}{|c|}{ Nature du pesticide contaminant** } \\
\hline & & & & & $\alpha \mathrm{HCH}$ & $\beta \mathrm{HCH}$ & $\gamma \mathrm{HCH}$ & $\mathrm{HE}$ & DIEL & DDT \\
\hline \multirow{4}{*}{ 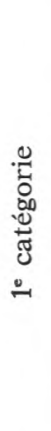 } & Concentrés & $\begin{array}{l}\text { Hiver } 70-71 \\
\text { Sept. } 1971 \\
\text { Mars } 1972\end{array}$ & $\begin{array}{l}33 \\
32 \\
21\end{array}$ & $\begin{array}{r}18 \\
16 \\
2\end{array}$ & $\begin{array}{r}12 \\
6 \\
2\end{array}$ & $\begin{array}{r}2 \\
3 \\
-\end{array}$ & $\begin{array}{r}1 \\
1 \\
-\end{array}$ & $\begin{array}{l}2 \\
4 \\
1\end{array}$ & $\begin{array}{r}9 \\
8 \\
-\end{array}$ & $\begin{array}{l}- \\
-\end{array}$ \\
\hline & Céréales & $\begin{array}{l}\text { Hiver } 70-71 \\
\text { Sept. } 1971 \\
\text { Mars } 1972\end{array}$ & $\begin{array}{l}73 \\
52 \\
48\end{array}$ & $\begin{array}{r}32 \\
6 \\
-\end{array}$ & $\begin{array}{r}27 \\
1 \\
-\end{array}$ & $\begin{array}{r}2 \\
2 \\
-\end{array}$ & $\begin{array}{r}3 \\
2 \\
-\end{array}$ & $\begin{array}{l}- \\
-\end{array}$ & $\begin{array}{r}6 \\
3 \\
-\end{array}$ & $\begin{array}{l}- \\
-\end{array}$ \\
\hline & Tourteau & $\begin{array}{l}\text { Hiver } 70-71 \\
\text { Sept. } 1971 \\
\text { Mars } 1972\end{array}$ & $\begin{array}{l}25 \\
19 \\
33\end{array}$ & $\begin{array}{r}15 \\
2 \\
5\end{array}$ & $\begin{array}{r}14 \\
1 \\
3\end{array}$ & $\begin{array}{r}9 \\
1 \\
-\end{array}$ & $\overline{1}$ & $\begin{array}{l}- \\
-\end{array}$ & $\begin{array}{r}10 \\
1 \\
3\end{array}$ & $\frac{1}{-}$ \\
\hline & Pulpe sèche & $\begin{array}{l}\text { Hiver } 70-71 \\
\text { Mars } 1972\end{array}$ & $\begin{array}{l}10 \\
12\end{array}$ & $\begin{array}{l}10 \\
12\end{array}$ & 1 & $\overline{6}$ & 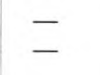 & $\begin{array}{l}10 \\
12\end{array}$ & $\begin{array}{r}8 \\
10\end{array}$ & 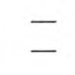 \\
\hline $\begin{array}{l}\dot{\tilde{J}} \\
\stackrel{\Xi}{\sim}\end{array}$ & Foins & $\begin{array}{l}\text { Hiver } 70-71 \\
\text { Sept. } 1971 \\
\text { Mars } 1972\end{array}$ & $\begin{array}{l}96 \\
44 \\
76\end{array}$ & $\begin{array}{l}58 \\
23 \\
29\end{array}$ & $\begin{array}{r}42 \\
7 \\
18\end{array}$ & $\begin{array}{r}3 \\
1 \\
-\end{array}$ & $\frac{-}{8}$ & $\begin{array}{r}10 \\
8 \\
9\end{array}$ & $\begin{array}{l}32 \\
13 \\
11\end{array}$ & $\begin{array}{l}- \\
-\end{array}$ \\
\hline & & & & & & & & & & \\
\hline
\end{tabular}




\begin{tabular}{|c|c|c|c|c|c|c|c|c|c|c|}
\hline \multirow{12}{*}{ 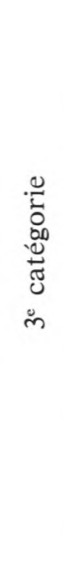 } & Choux - colza & Hiver $70-71$ & 58 & 7 & 2 & & & 4 & & \\
\hline & Betteraves & Sept. 1971 & 9 & 5 & - & 1 & 1 & - & 3 & - \\
\hline & (Racines et collets) & Mars 1972 & 33 & 16 & - & 3 & - & 8 & 15 & - \\
\hline & Fourrages verts & Hiver $70-71$ & 7 & 3 & $\overline{1 .}$ & - & - & 3 & - & - \\
\hline & & Sept. 1971 & $\pi 1$ & 22 & 15 & 3 & - & 12 & 3 & - \\
\hline & Maïs vert & Sept. 1971 & 19 & - & - & - & - & - & - & - \\
\hline & Sorgho & Sept. 1971 & 2 & 1 & 1 & 1 & - & - & 1 & - \\
\hline & Ensilages divers & Hiver $70-71$ & 11 & 3 & 2 & - & - & 1 & 1 & - \\
\hline & & Sept. 1971 & 13 & 3 & $\overline{1}$ & $\overline{5}$ & 1 & - & 2 & - \\
\hline & Ensilages de maïs & & & & 3 & & & & & \\
\hline & & Sept. 1971 & 3 & 1 & - & - & - & 1 & 1 & - \\
\hline & & Mars 1972 & 23 & 14 & - & 3 & - & 4 & 14 & - \\
\hline
\end{tabular}

* "Echantillon contaminé " = tout échantillon dans lequel la quantité dépistée d'au moins un des pesticides analysés est égale ou supérieure au seuil indiqué dans le tableau 1, pour la catégorie d'aliment correspondante.

** Fréquence d'apparition de chacun des pesticides dans les échantillons contaminés (*). 
- par des erreurs d'emploi de produits antiparasitaires peu dangereux à dose normale, ou bien par l'utilisation de produits, jusqu'alors autorisés, possédant une forte rémanence et s'accumulant à un point quelconque du cycle de production.

Du fait d'interdictions successives, plusieurs des causes de contamination sont passées au second plan. En fin d'enquête, il s'avère que l'alimentation devient la cause principale de la contamination du lait. C'est pourquoi, nous étudierons successivement :

- l'évolution de la contamination aliment par aliment, au cours de l'enquête,

- l'évolution de la contamination du lait en $\mathrm{HCH}$, dieldrine et heptachlore-époxyde dans les différentes régions.

\section{IV. - LA CONTAMINATION DES ALIMENTS}

Nous avons pu établir le tableau suivant à partir des données de la recherche et de l'ensemble des résultats de l'enquête (tab. 1).

Prenons quelques exemples : nous savons que pour obtenir $0,10 \mathrm{ppm}$ de $\alpha \mathrm{HCH}$ dans la matière grasse du lait, il faut $0,080 \mathrm{ppm}$ de ce résidu dans la matière sèche totale de la ration; en l'absence de toute autre contamination, nous obtiendrons les $0,10 \mathrm{ppm}$ avec :

- $3 \mathrm{~kg}$ de concentré à $0,030 \mathrm{ppm}$, ou bien :

- $7 \mathrm{~kg}$ de foin à $0,015 \mathrm{ppm}$, ou encore :

- tout aliment frais à 0,010 ppm.

Trois séries de prélèvements ont été faites sur les aliments :

- hiver 1970-1971,

- septembre 1971,

- mars 1972.

Afin de constater s'il y a eu une évolution de la contamination entre les deux hivers, l'ensemble des résultats aliment par aliment est rassemblé dans le tableau 2.

L'examen du tableau 2 permet d'énoncer les remarques suivantes :

Aliments de la première catégorie :

- La contamination des aliments de cette catégorie est en général en évolution favorable à l'exception des pulpes. La diminution est surtout sensible au niveau des résidus des isomères $\alpha$ et $\beta$ de l'HCH.

- L'arrêt de l'utilisation des insecticides organochlorés pour le traitement des locaux de stockage a eu un effet direct sur l'évolution de la contamination; les échantillons de cette catégorie sont plus rarement contaminés. 
- L'introduction des pulpes dans l'alimentation est d'autant plus catastrophique que nous avons pu constater une contamination moyenne en heptachlore-époxyde de cet aliment plus importante le deuxième hiver $(0,40 \mathrm{ppm})$ que le premier $(0,25 \mathrm{ppm})$.

Aliments de la deuxième et de la troisième catégorie :

- L'énorme quantité de fourrage grossier absorbé chaque jour par une vache laitière donne une idée du risque que fait courir une contamination apparemment modeste de cet aliment.

- La contamination des foins est de ce fait relativement importante. Si l'on peut constater une diminution des résidus de $\mathrm{HCH}$, le fait du renouvellement des cultures surtout dans l'ouest, provoque la présence non négligeable de résidus d'heptachlore-époxyde et de dieldrine.

- Dans les aliments humides, nous retrouvons principalement des résidus de $\alpha \mathrm{HCH}$, d'heptachlore-époxyde et de dieldrine. En ce qui concerne les résidus de $\alpha \mathrm{HCH}$, il s'agit d'une utilisation intempestive de cet insecticide. Pour les autres résidus, la cause de leur présence est multiple :

- fourrages en culture dérobée,

- prairies cultivées dans un assolement comprenant du maïs grain ou de la betterave sucrière,

- implantation récente d'une nouvelle culture après retournement d'une prairie et traitement du sol avec un insecticide organochloré.

\section{V. - L'EVOLUTION DE LA CONTAMINATION DU LAIT DANS LES DIFFERENTES REGIONS DE L'ENQUETE}

Les exploitations représentant l'échantillon ont été rassemblées en zones :

- Zone de cultures industrielles du Nord et de l'Est de la France : Nord, Pas-de-Calais, Marne (C.I.) : 9 producteurs.

- Zone herbagère limitrophe de la zone précédente : Aisne, Meuse, Moselle, Haute-Marne, Aube (N.E.) : 14 producteurs.

- Zone du Centre-Est (C.E.) : région Rhône-Alpes et département du Jura : 19 producteurs.

- Zone de l'Ouest (O.) : les pays de la Loire et les départements de l'Ille-et-Vilaine et des Deux-Sèvres : 28 producteurs. ducteurs.

- Zone du Sud-Ouest (S.O.) : région Midi-Pyrénées : 12 pro-

- Zone brebis (B.) : département de l'Aveyron : 7 producteurs. 
TABLEAU 3. - Evolution de la contamination du lait : résidus de $\mathrm{HCH}^{* *}$

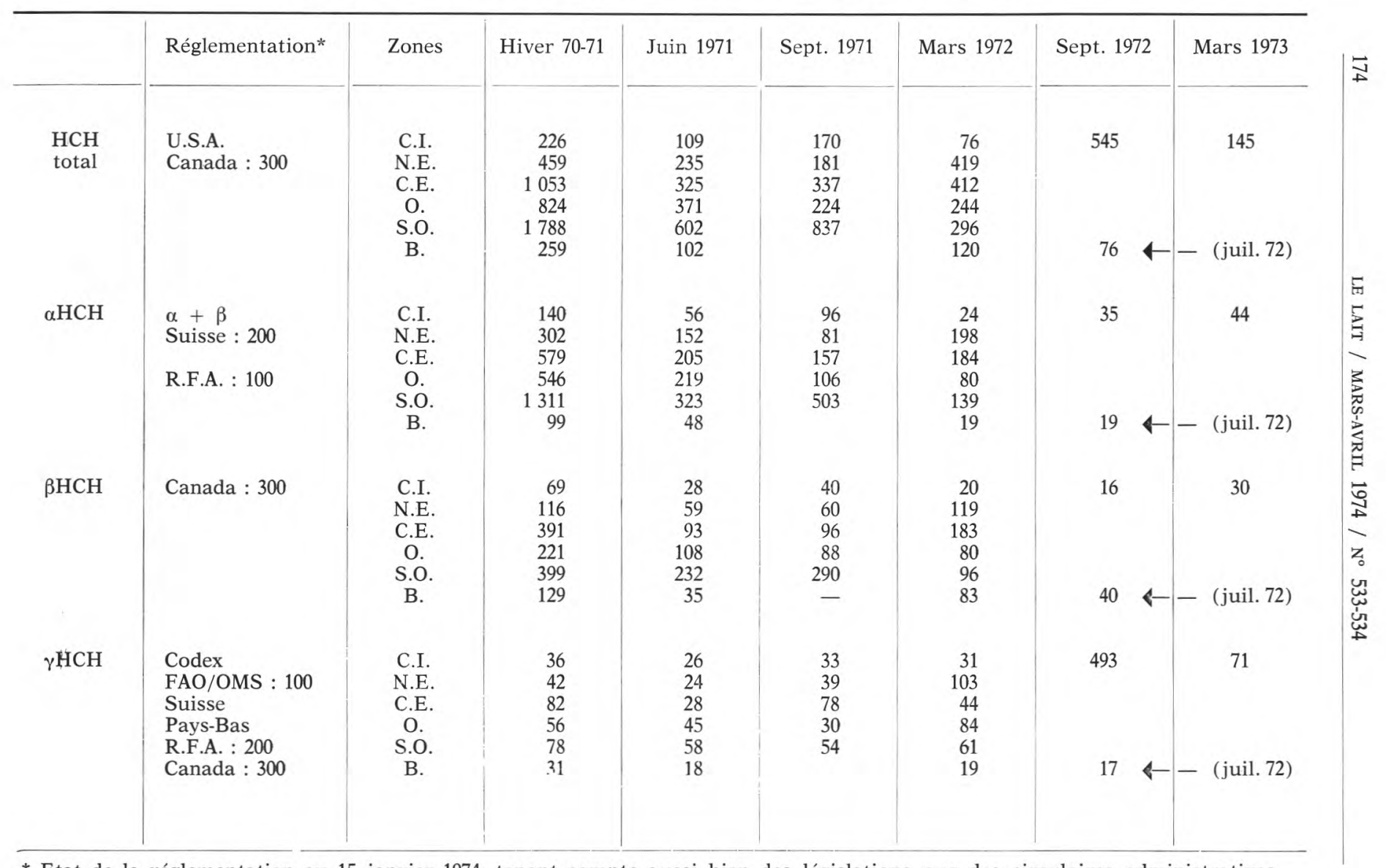

* Etat de la réglementation au 15 janvier 1974, tenant compte aussi bien des législations que des circulaires administratives.

** Résultats exprimés en ppb $=u \mathrm{~g}$ par $\mathrm{kg}$ rapporté à la M.G. 
Dans les tableaux ci-après, sont rassemblées les moyennes des résultats de chaque producteur de la zone considérée pour chacun des 4 prélèvements principaux.

Remaroues : L'état de la réglementation présentée est valable au 15 janvier 1974. Il tient compte aussi bien des législations que des circulaires administratives.

Chaque zone est représentée par son sigle.

Les quantités de résidus sont exprimées en $\mathrm{ppb}$ ( $\mu \mathrm{g}$ par $\mathrm{kg}$ rapporté à la matière grasse).

REMARQue IMPORTANTE : Etant donné la constitution de l'échantillon, les résultats énoncés ci-après indiquent généralement une contamination plus importante que celle existant effectivement.

\section{a) Evolution de la contamination du lait par des résidus de $\mathbf{H C H}$ (tab. 3)}

On constate une diminution importante de la contamination d'un hiver sur l'autre. Dès mars 1972, les tolérances nord-américaines sont approchées, mais pour certaines régions, la norme allemande (100 ppb) ne l'est pas.

Du fait de l'interdiction du $\mathrm{HCH}$ pour la désinsectisation des étables et de tous locaux servant au logement des animaux domestiques ou à la préparation de leur nourriture, la présence de ce résidu ne peut que diminuer ces prochaines années.

On peut constater une dangereuse stabilisation des résidus de $\gamma \mathrm{HCH}$ due principalement aux traitements vétérinaires. Mais dans les régions de cultures industrielles, la présence de ce résidu a doublé entre les hivers $71-72$ et $72-73$ et cela, du fait du seul traitement des sols.

\section{b) Evolution de la contamination du lait par des résidus de HCB} (tab. 4)

Aucune conclusion ne peut être tirée de ce tableau, les causes de la présence de ce résidu dans le lait étant encore à l'étude. Cependant, aucun résultat n'atteint le seuil des $300 \mathrm{ppb}$.

\section{c) Evolution de la contamination du lait par des résidus d'hepta- chlore-époxyde (tab. 5)}

Dans les régions de cultures industrielles, l'escalade de la contamination est catastrophique. Ceci correspond à la rétrocession de pulpes pour l'alimentation des animaux. Dans ces zones, les sols sont presque saturés en résidus d'heptachlore-époxyde. Si bien que, vu la rémanence de ce produit, les laits n'approcheront les seuils précités pas avant 4 ou 5 ans. 
TABLEAU 4. - Résidus de HCB*

\begin{tabular}{|c|c|c|c|c|c|c|c|}
\hline Réglementation* & Zones & Hiver 70-71 & Juin 1971 & Sept. 1971 & Mars 1972 & Sept. 1972 & Mars 1973 \\
\hline $\begin{array}{l}\text { Codex FAO/OMS } \\
\text { U.S.A. } \\
\text { Suisse : } 300 \\
\text { Canada : } 100 \\
\text { R.F.A. : } 500\end{array}$ & $\begin{array}{l}\text { C.I. } \\
\text { N.E. } \\
\text { C.E. } \\
\text { O. } \\
\text { S.O. } \\
\text { B. }\end{array}$ & $\begin{array}{r}53 \\
60 \\
84 \\
51 \\
41 \\
109\end{array}$ & $\begin{array}{l}48 \\
36 \\
52 \\
60 \\
46 \\
25\end{array}$ & $\begin{array}{l}33 \\
24 \\
57 \\
37 \\
98 \\
-\end{array}$ & $\begin{array}{l}34 \\
39 \\
59 \\
40 \\
41 \\
36\end{array}$ & 90 & 86 \\
\hline \multicolumn{8}{|c|}{ TABLEAU 5. - Résidus d'heptachlore-époxyde* } \\
\hline Réglementation* & Zones & Hiver 70-71 & Juin 1971 & Sept. 1971 & Mars 1972 & Sept. 1972 & Mars 1973 \\
\hline $\begin{array}{l}\text { Codex FAO/OMS } \\
\text { R.F.A. : } 150 \\
\text { Suisse } \\
\text { Pays-Bas : } 125 \\
\text { U.S.A. } \\
\text { Canada : } 300\end{array}$ & $\begin{array}{c}\text { C.I. } \\
\text { N.E. } \\
\text { C.E. } \\
\text { O. } \\
\text { S.O. } \\
\text { B. }\end{array}$ & $\begin{array}{r}581 \\
205 \\
94 \\
106 \\
53 \\
19\end{array}$ & $\begin{array}{r}441 \\
112 \\
39 \\
115 \\
47 \\
53\end{array}$ & $\begin{array}{r}267 \\
45 \\
24 \\
70 \\
33\end{array}$ & $\begin{array}{r}886 \\
285 \\
62 \\
109 \\
35 \\
10\end{array}$ & 498 & - (juil. 72) \\
\hline \multicolumn{8}{|c|}{ TABLEAU 6. - Résidus de dieldrine* } \\
\hline Réglementation* & Zones & Hiver 70-71 & Juin 1971 & Sept. 1971 & Mars 1972 & Sept. 1972 & Mars 1973 \\
\hline $\begin{array}{l}\text { Codex FAO/OMS } \\
\text { R.F.A. : } 150 \\
\text { Canada : } 100 \\
\text { Suisse } \\
\text { Pays-Bas : } 125 \\
\text { U.S.A. : } 300\end{array}$ & $\begin{array}{c}\text { C.I. } \\
\text { N.E. } \\
\text { C.E. } \\
\text { O. } \\
\text { S.O. } \\
\text { B. }\end{array}$ & $\begin{array}{r}129 \\
108 \\
133 \\
46 \\
69 \\
37\end{array}$ & $\begin{array}{r}136 \\
103 \\
49 \\
118 \\
88 \\
19\end{array}$ & $\begin{array}{l}67 \\
36 \\
42 \\
49 \\
58\end{array}$ & $\begin{array}{r}104 \\
39 \\
27 \\
51 \\
48 \\
10\end{array}$ & 23 & - (juil. 72) \\
\hline
\end{tabular}


Dans la zone limitrophe, on constate une augmentation de la contamination d'un hiver sur l'autre. Cette augmentation est principalement due à l'importation de pulpes sèches. Un arrêt de cette importation permettrait d'atteindre assez rapidement les seuils demandés.

Dans les autres zones, cette contamination est récente et principalement due au retournement des prairies. Il serait donc possible d'atteindre aussi rapidement les seuils demandés.

\section{d) Evolution de la contamination du lait par les résidus de diel- drine (tab. 6)}

La contamination semble contenue dans les seuils ; cependant, les remarques énoncées à propos des résidus d'heptachlore-époxyde restent valables dans la mesure où peu de producteurs de l'échantillon ont utilisé cet insecticide.

\section{VI. - CONCLUSION}

\section{a) Contamination des aliments}

La contamination en résidus d'heptachlore-époxyde et de dieldrine de la ration de base des vaches laitières est relativement importante à la fin de l'hiver 1971-1972. Elle semble être due à l'évolution actuelle de l'élevage qui tend à remplacer les éléments traditionnels, par d'autres tels les ensilages de maïs, les herbes cultivées... Ceci demande un retournement des prairies naturelles et généralement un traitement insecticide des sols à ce moment.

L'interdiction de l'aldrine, de la dieldrine et de l'heptachlore en agriculture devrait donc favoriser la diminution des résidus de ces produits dans l'alimentation, à partir de la campagne 1973-1974.

Quant aux résidus de $\mathrm{HCH}$, ils semblent en voie de disparition au niveau de l'alimentation.

\section{b) Evolution de la contamination des laits}

Quel que soit le pesticide considéré, l'époque où le niveau de contamination est le plus élevé, se situe en hiver et surtout à la fin de l'hiver, c'est-à-dire au moment où les animaux sont à l'étable ou en stabulation.

La contamination dépend largement des pratiques alimentaires. Il n'est pas étonnant en conséquence que des régions ayant une certaine homogénéité dans le type d'alimentation, présentent des contaminations semblables.

On constate d'un hiver sur l'autre une diminution importante mais non encore suffisante, de la contamination du lait par des 
résidus de $\mathrm{HCH}$. Si l'interdiction d'utiliser le $\mathrm{HCH}$ en agriculture est respectée, la présence de ce résidu ne peut que diminuer ces prochaines années.

On constate aussi une dangereuse stabilisation des résidus de lindane due principalement aux traitements vétérinaires, mais aussi aux traitements inconsidérés des sols.

On observe enfin une escalade de la contamination par des résidus d'heptachlore-époxyde : la situation est catastrophique dans la région des cultures industrielles; elle est loin d'être alarmante dans les autres régions. Si l'interdiction d'utiliser les cyclodiènes en agriculture est respectée, il sera possible d'atteindre très rapidement les seuils demandés, excepté dans la zone des cultures betteravières.

\section{S u m m ar y}

A survey had been made by the Milk Department of the Institute for Beef and Dairy Cattle (I.T.E.B. Paris) in view of standing out the most frequent process of milk pollution by organochlorine compounds, for the ratio of their concentration in milk happens to be much higher than the upper rates generally admitted in that food.

The information stated in this study was collected from data available on french farms. The present work may be far from being a statistical report. Its main purpose was rather to make a most detailed analysis of typical samples allocated to areas spread over all the french territory.

On account of successive prohibitions restraining the use of certain chemicals (the development of the survey contributed to bring about such a change) contamination sources lockened down, e.g. whitening of buildings for dairy cattle, and so for that in 1972 pollution was mainly in touch with animal feeding.

Further research on each type of feed used for dairy cattle made it possible to draw conclusions allowing to know wether the change in regulations concerning the use of organochlorine compounds in agriculture were well advised or not.

Now the point about the contamination of milk in the future... In fact, the concentration of B.H.C. (H.C.H.) residues found in milk has been decreasing from year's end to year's end. On the other hand the situation is still unsatisfactory in certain areas because of the high ratio of pesticides found in milk, when comparing these results with those established for foreign products.

As far as Heptachlor epoxyde residues are concerned, the contamination evolved into a great disaster, particulary in agricultural regions devoted to the production of industrial crops, as far as contaminated beet pulps are being for feeding dairy cattle. 


\section{Bibliographie}

1. Hascoet (M.). - Les résidus de pesticides dans les produits laitiers. Bulletin Technique d'Information, août-septembre 1970, p. 521-534.

2. Luquet (F. M.). - Thèse : Contribution à la connaissance de la pollution des laits en R.P.O., 1973, Université de Caen.

3. Richou-Bac (L.). - Les résidus de pesticides dans les viandes, les graisses animales et végétales. Bulletin Technique d'Information, août-septembre 1970, p. $535-546$.

\section{Publications de la section lait de l'I.T.E.B.}

1. Plaquette établie en relation avec la «Protection des plantes et environnement ». Prix : 1 F l'unité, 0,50 F par 100. Comment éviter la contamination du lait par les produits antiparasitaires : Méthodes de travail. Produits de remplacement.

2. Compte rendu établi par Hervé Mahieu (prix $10 \mathrm{~F}$ ). Les sources de contamination du lait par des résidus de pesticides organochlorés : Résultats d'une enquête effectée par la section lait de l'I.T.E.B. au cours des années 1970, 1971, 1972. Rappel de la législation internationale.

Ces brochures peuvent être obtenues auprès de la Société Technipel, 149, rue de Bercy - 75579 Paris cedex 12. Tél. : 346-12-20. 